\title{
The joint essential numerical range, compact perturbations, and the Olsen problem
}

\author{
by \\ Vladimír MÜller (Praha) \\ Dedicated to Professor Muneo Chō on his 60th birthday
}

\begin{abstract}
Let $T_{1}, \ldots, T_{n}$ be bounded linear operators on a complex Hilbert space $H$. Then there are compact operators $K_{1}, \ldots, K_{n} \in B(H)$ such that the closure of the joint numerical range of the $n$-tuple $\left(T_{1}-K_{1}, \ldots, T_{n}-K_{n}\right)$ equals the joint essential numerical range of $\left(T_{1}, \ldots, T_{n}\right)$. This generalizes the corresponding result for $n=1$.

We also show that if $S \in B(H)$ and $n \in \mathbb{N}$ then there exists a compact operator $K \in B(H)$ such that $\left\|(S-K)^{n}\right\|=\left\|S^{n}\right\|_{e}$. This generalizes results of C. L. Olsen.
\end{abstract}

1. Introduction. Let $T$ be a bounded linear operator acting on a complex Hilbert space $H$. The properties of $T$ can be frequently improved by adding to it a compact perturbation. For example:

- [S] there exists a compact operator $K_{1} \in B(H)$ such that

$$
\sigma\left(T+K_{1}\right)=\sigma_{W}(T),
$$

where $\sigma_{W}(T)=\bigcap\{\sigma(T+L): L \in B(H)$ compact $\}$ denotes the Weyl spectrum of $T$;

- $\left[\mathrm{CSSW}\right.$ there exists a compact operator $K_{2}$ such that $\overline{W\left(T+K_{2}\right)}=$ $W_{e}(T)$, where $W$ denotes the numerical range and $W_{e}$ the essential numerical range;

- $[\mathrm{OP}]$ if $p$ is a polynomial and $p(T)$ is compact, then there exists a compact operator $K_{3}$ such that $p\left(T+K_{3}\right)=0$.

The present paper studies variations of the second and third result mentioned above.

In the next section we generalize the second result to $n$-tuples of operators.

2010 Mathematics Subject Classification: Primary 47A12; Secondary 47A13.

Key words and phrases: joint essential numerical range, joint numerical range, compact perturbation, Olsen's problem. 
In Section 3 we study the following problem of C. L. Olsen which is still open: if $S \in B(H)$ and $p$ a polynomial, does there exist a compact operator $L$ such that $\|p(S+L)\|=\|p(S)\|_{e}$ ?

A positive answer is known only for some special polynomials. In $[\mathrm{O}]$ it was proved for $p(z)=z, z^{2}, z^{3}$. In [CLSW] a positive answer was given for all linear polynomials.

We improve the result of $[\mathrm{O}]$ and give a positive answer for all powers $p(z)=z^{n}$.

2. Joint numerical range and compact perturbations. Let $T=$ $\left(T_{1}, \ldots, T_{n}\right)$ be an $n$-tuple of operators on a complex Hilbert space $H$. The joint numerical range $W(T)$ is defined by

$$
W(T)=\left\{\left(\left\langle T_{1} x, x\right\rangle, \ldots,\left\langle T_{n} x, x\right\rangle\right): x \in H,\|x\|=1\right\} .
$$

It is well known that the numerical range $W(T)$ is convex for $n=1$ but not convex in general for $n \geq 2$.

Apart from the (spatial) numerical range $W(T)$ it is also possible to define an algebraic numerical range. Let $\mathcal{A}$ be a Banach algebra with unit $e$, let $a=\left(a_{1}, \ldots, a_{n}\right) \in \mathcal{A}^{n}$. The joint algebraic numerical range $V(a, \mathcal{A})$ is defined by

$$
V(a, \mathcal{A})=\left\{\left(f\left(a_{1}\right), \ldots, f\left(a_{n}\right)\right): f \in \mathcal{A}^{*},\|f\|=1=f(e)\right\} .
$$

It is well known that $V(a, \mathcal{A})$ is always a compact convex subset of $\mathbb{C}^{n}$; see [BD1, p. 24].

For $n$-tuples of Hilbert space operators the spatial and algebraic numerical ranges are closely connected. Denote by $B(H)$ the algebra of all bounded linear operators on a Hilbert space $H$. By $\overline{c o n v} M$ we denote the closed convex hull of a set $M$.

Theorem 1. Let $T=\left(T_{1}, \ldots, T_{n}\right) \in B(H)^{n}$. Then

$$
V(T, B(H))=\overline{\mathrm{conv}} W(T) .
$$

Proof. The statement is well known for $n=1$ (see [BD1, p. 83]). For $n \geq 2$ it is more or less folklore. For the sake of convenience we briefly indicate the proof.

Clearly, $W(T) \subset V(T, B(H))$. Since $V(T, B(H))$ is closed and convex, we have $\overline{\operatorname{conv}} W(T) \subset V(T, B(H))$.

Let $z=\left(z_{1}, \ldots, z_{n}\right) \in \mathbb{C}^{n} \backslash \overline{\operatorname{conv}} W(T)$. Then there are $\alpha_{1}, \ldots, \alpha_{n} \in \mathbb{C}$ such that 


$$
\begin{aligned}
\operatorname{Re} \sum_{j=1}^{n} \alpha_{j} z_{j} & >\sup \left\{\operatorname{Re} \sum_{j=1}^{n} \alpha_{j} \lambda_{j}:\left(\lambda_{1}, \ldots, \lambda_{n}\right) \in W(T)\right\} \\
& =\sup \left\{\operatorname{Re}\left\langle\sum_{j=1}^{n} \alpha_{j} T_{j} x, x\right\rangle: x \in H,\|x\|=1\right\} \\
& =\sup \left\{\operatorname{Re} \mu: \mu \in W\left(\sum_{j=1}^{m} \alpha_{j} T_{j}\right)\right\} \\
& =\sup \left\{\operatorname{Re} \mu: \mu \in V\left(\sum_{j=1}^{n} \alpha_{j} T_{j}, B(H)\right)\right\} \\
& =\sup \left\{\operatorname{Re} \sum_{j=1}^{n} \alpha_{j} f\left(T_{j}\right): f \in B(H)^{*},\|f\|=1=f(I)\right\} \\
& =\sup \left\{\operatorname{Re} \sum_{j=1}^{n} \alpha_{j} \lambda_{j}:\left(\lambda_{1}, \ldots, \lambda_{n}\right) \in V(T, B(H))\right\} .
\end{aligned}
$$

Hence $\left(z_{1}, \ldots, z_{n}\right) \notin V(T, B(H))$ and $V(T, B(H))=\overline{\mathrm{conv}} W(T)$.

Let $H$ be an infinite-dimensional Hilbert space. The joint essential numerical range $W_{e}(T)$ of an $n$-tuple $T=\left(T_{1}, \ldots, T_{n}\right) \in B(H)^{n}$ is the set of all $\left(\lambda_{1}, \ldots, \lambda_{n}\right) \in \mathbb{C}^{n}$ such that there exists an orthonormal sequence $\left(x_{k}\right) \subset H$ with

$$
\lambda_{j}=\lim _{k \rightarrow \infty}\left\langle T_{j} x_{k}, x_{k}\right\rangle \quad(j=1, \ldots, n) .
$$

The essential numerical range for $n=1$ was introduced and studied in [FSW]. The joint essential numerical range was studied e.g. in [TC], [B], [LP].

Although $W(T)$ is not convex in general for $n \geq 2$, it was shown in [LP] that $W_{e}(T)$ is always convex. In fact, $W_{e}(T)$ is equal to the algebraic numerical range of the corresponding classes in the Calkin algebra $B(H) / \mathcal{K}(H)$, where $\mathcal{K}(H)$ denotes the ideal of all compact operators on $H$. Denote by $\pi: B(H) \rightarrow B(H) / \mathcal{K}(H)$ the canonical projection. As usual, we write $\pi(T)=\left(\pi\left(T_{1}\right), \ldots, \pi\left(T_{n}\right)\right)$.

We summarize the basic properties of the joint essential numerical range $W_{e}(T)$ in the following theorem:

THEOREM 2. Let $H$ be an infinite-dimensional Hilbert space and let $T=$ $\left(T_{1}, \ldots, T_{n}\right) \in B(H)^{n}$. Then $W_{e}(T)$ is a compact convex subset of $\mathbb{C}^{n}$ and

$$
\begin{aligned}
W_{e}(T) & =\bigcap_{K \in \mathcal{K}(H)^{n}} \overline{W(T+K)}=\bigcap_{K \in \mathcal{K}(H)^{n}} \overline{\operatorname{conv}} W(T+K) \\
& =V(\pi(T), B(H) / \mathcal{K}(H)) .
\end{aligned}
$$

Proof. The first two equalities were proved in $[\mathrm{LP}]$. They also imply the compactness and convexity of $W_{e}(T)$. 
If $f \in(B(H) / \mathcal{K}(H))^{*}$ and $\|f\|=1=f(I+\mathcal{K}(H))$ then $f \circ \pi \in B(H)^{*}$ and $\|f \circ \pi\|=1=(f \circ \pi)(I)$. Moreover, $f \circ \pi$ annihilates compact operators. So

$$
V(\pi(T), B(H) / \mathcal{K}(H)) \subset V(T+K, B(H))
$$

for all $K \in \mathcal{K}(H)^{n}$. Hence

$$
\begin{aligned}
V(\pi(T), B(H) / \mathcal{K}(H)) & \subset \bigcap_{K \in \mathcal{K}(H)^{n}} V(T+K, B(H)) \\
& =\bigcap_{K \in \mathcal{K}(H)^{n}} \overline{\operatorname{conv}} W(T+K)=W_{e}(T) .
\end{aligned}
$$

Conversely, let $\lambda=\left(\lambda_{1}, \ldots, \lambda_{n}\right) \in W_{e}(T)$, i.e., there exists an orthonormal sequence $\left(x_{k}\right) \subset H$ such that $\lambda_{j}=\lim _{k \rightarrow \infty}\left\langle T_{j} x_{k}, x_{k}\right\rangle$ for all $j=$ $1, \ldots, n$. Let LIM be any Banach limit. Define $f \in B(H)^{*}$ by $f(S)=$ $\operatorname{LIM}_{k \rightarrow \infty}\left\langle S x_{k}, x_{k}\right\rangle(S \in B(H))$. Then $f$ annihilates all compact operators and so it induces a functional $\tilde{f} \in(B(H) / \mathcal{K}(H))^{*}$. Clearly, $\|\tilde{f}\|=1=$ $\tilde{f}(I+\mathcal{K}(H))$ and $\tilde{f}(\pi(T))=\lambda$. Hence $\lambda \in V(\pi(T), B(H) / \mathcal{K}(H))$.

The main result of this section is that for each $n$-tuple $\left(T_{1}, \ldots, T_{n}\right)$ of Hilbert space operators there exists an $n$-tuple of compact operators $\left(K_{1}, \ldots, K_{n}\right)$ such that $W_{e}\left(T_{1}, \ldots, T_{n}\right)=W\left(T_{1}-K_{1}, \ldots, T_{n}-K_{n}\right)^{-}$. This improves the results of $[\mathrm{LP}]$ mentioned in Theorem 2.

For $n=1$ the existence of the optimal compact perturbation was proved in [CSSW]. We use the operator-theoretical method of [CLSW] (however, this method is only sketched there in a not very clear way, the paper [CLSW] uses mainly another method based on the notion of $M$-ideals).

REMARK 3. In fact, the joint essential numerical range $W_{e}(T)$ was studied in $[\mathrm{LP}]$ only for $n$-tuples of selfadjoint operators. However, every operator $S \in B(H)$ can be written as $S=\operatorname{Re} S+i \operatorname{Im} S$, where $\operatorname{Re} S=\frac{1}{2}\left(S+S^{*}\right)$ and $\operatorname{Im} S=\frac{1}{2 i}\left(S-S^{*}\right)$ are selfadjoint operators.

Any $n$-tuple $T=\left(T_{1}, \ldots, T_{n}\right) \in B(H)^{n}$ can be identified with the $(2 n)$ tuple

$$
\left(\operatorname{Re} T_{1}, \operatorname{Im} T_{1}, \ldots, \operatorname{Re} T_{n}, \operatorname{Im} T_{n}\right)
$$

of selfadjoint operators and the joint essential numerical range $W_{e}(T) \subset \mathbb{C}^{n}$ can be identified with $W_{e}\left(\operatorname{Re} T_{1}, \operatorname{Im} T_{1}, \ldots, \operatorname{Re} T_{n}, \operatorname{Im} T_{n}\right) \subset \mathbb{R}^{2 n}$. So all the statements concerning $W_{e}(T)$ can be reduced to the corresponding statements for tuples of selfadjoint operators.

An important property of selfadjoint operators is that their numerical range is real. The next proposition characterizes the numerical range of tuples of selfadjoint operators.

Proposition 4. Let $S=\left(S_{1}, \ldots, S_{n}\right)$ be an $n$-tuple of selfadjoint operators on a Hilbert space $H$. Let $z=\left(z_{1}, \ldots, z_{1}\right) \in \mathbb{R}^{n}$. Then: 
(i) $z \in V(S, B(H))$ if and only if

$$
\left|\sum_{j=1}^{n} \alpha_{j} z_{j}+\lambda\right| \leq\left\|\sum_{j=1}^{n} \alpha_{j} S_{j}+\lambda\right\|
$$

for all $\alpha_{1}, \ldots, \alpha_{n}, \lambda \in \mathbb{R}$.

(ii) $z \in W_{e}(S)$ if and only if

$$
\left|\sum_{j=1}^{n} \alpha_{j} z_{j}+\lambda\right| \leq\left\|\sum_{j=1}^{n} \alpha_{j} S_{j}+\lambda\right\|_{e}
$$

for all $\alpha_{1}, \ldots, \alpha_{n}, \lambda \in \mathbb{R}$.

Proof. Let $z \in V(S, B(H))$. Then there exists $f \in B(H)^{*}$ such that $\|f\|=1=f(I)$ and $f\left(S_{j}\right)=z_{j}(j=1, \ldots, n)$. Let $\alpha_{1}, \ldots, \alpha_{n}, \lambda \in \mathbb{R}$. Then

$$
\left|\sum_{j=1}^{n} \alpha_{j} z_{j}+\lambda\right|=\left|f\left(\sum_{j=1}^{n} \alpha_{j} S_{j}+\lambda I\right)\right| \leq\left\|\sum_{j=1}^{n} \alpha_{j} S_{j}+\lambda\right\| .
$$

Conversely, let $\left|\sum_{j=1}^{n} \alpha_{j} z_{j}+\lambda\right| \leq\left\|\sum_{j=1}^{n} \alpha_{j} S_{j}+\lambda\right\|$ for all $\alpha_{1}, \ldots, \alpha_{n}, \lambda$ $\in \mathbb{R}$. Let $\mathcal{A}$ be the real subspace of $B(H)$ generated by $S_{1}, \ldots, S_{n}, I$. Let $f$ : $\mathcal{A} \rightarrow \mathbb{R}$ be the real functional defined by $f\left(\sum_{j=1}^{n} \alpha_{j} S_{j}+\lambda I\right)=\sum_{j=1}^{n} \alpha_{j} z_{j}+\lambda$. Then $\|f\| \leq 1$ and $f(I)=1$. So $\|f\|=1$ and $f\left(S_{j}\right)=z_{j}(j=1, \ldots, n)$.

By the Hahn-Banach theorem there exists a functional $\tilde{f}: B(H) \rightarrow \mathbb{R}$ extending $f$ such that $\|\tilde{f}\|=\|f\|=1$. Let $g \in B(H)^{*}$ be the complex functional defined by $g(V)=\tilde{f}(V)-i \tilde{f}(i V)(V \in B(H))$. Then $\|g\|=\|\tilde{f}\|=1$ and $\tilde{f}=\operatorname{Re} g($ see $[\mathrm{BD} 2$, p. 3]). We have $|g(I)| \leq 1$ and $\operatorname{Re} g(I)=\tilde{f}(I)=1$, so $g(I)=1$. Thus $g\left(S_{1}, \ldots, S_{n}\right) \in V(S, B(H))$.

Moreover, $V(S, B(H)) \subset \prod_{j=1}^{n} V\left(S_{j}, B(H)\right) \subset \mathbb{R}^{n}$. So

$$
\begin{aligned}
\left(z_{1}, \ldots, z_{n}\right) & =\left(\tilde{f}\left(S_{1}\right), \ldots, \tilde{f}\left(S_{n}\right)\right)=\left(\operatorname{Re} g\left(S_{1}\right), \ldots, \operatorname{Re} g\left(S_{n}\right)\right) \\
& =\left(g\left(S_{1}\right), \ldots, g\left(S_{n}\right)\right) \in V(S, B(H)) .
\end{aligned}
$$

Part (ii) can be proved similarly using $W_{e}(S)=V(\pi(S), B(H) / \mathcal{K}(H))$.

Lemma 5. Let $H$ be a Hilbert space and let $S \in B(H), x \in H,\|x\|=1$, $t \geq 5\|S\|$. Then

$$
|\|(S+t) x\|-t-\operatorname{Re}\langle S x, x\rangle| \leq \frac{2\|S\|^{2}}{t} .
$$

Proof. We have

$$
\begin{aligned}
\|(S+t) x\| & \left.=(\| S+t) x \|^{2}\right)^{1 / 2}=\left(t^{2}+2 t \operatorname{Re}\langle S x, x\rangle+\|S x\|^{2}\right)^{1 / 2} \\
& =t(1+s)^{1 / 2}
\end{aligned}
$$


where $s=2 \operatorname{Re}\langle S x, x\rangle / t+\|S x\|^{2} / t^{2}$. Then

$$
s \leq \frac{2\|S\|}{t}+\frac{\|S\|^{2}}{t^{2}} \leq \frac{11\|S\|}{5 t} \leq \frac{1}{2} .
$$

It is easy to verify that for $s \leq 1 / 2$ we have

So

$$
1+\frac{s}{2}-\frac{s^{2}}{4} \leq(1+s)^{1 / 2} \leq 1+\frac{s}{2} .
$$

$$
\begin{aligned}
|\|(S+t) x\|-t-\operatorname{Re}\langle S x, x\rangle| & =\left|t(1+s)^{1 / 2}-t-\operatorname{Re}\langle S x, x\rangle\right| \\
& \leq\left|t(1+s)^{1 / 2}-t-\frac{t s}{2}\right|+\left|\frac{t s}{2}-\operatorname{Re}\langle S x, x\rangle\right| \\
& \leq t\left|(1+s)^{1 / 2}-1-\frac{s}{2}\right|+\frac{\|S x\|^{2}}{2 t} \\
& \leq \frac{t s^{2}}{4}+\frac{\|S\|^{2}}{2 t} \leq \frac{\|S\|^{2}}{t}\left(\frac{11^{2}}{4 \cdot 5^{2}}+\frac{1}{2}\right) \leq \frac{2\|S\|^{2}}{t} .
\end{aligned}
$$

For $S \in B(H)$ denote by $\|S\|_{e}$ the essential norm of $S,\|S\|_{e}=$ $\inf \{\|S+L\|: L \in \mathcal{K}(H)\}$. For a subspace $M \subset H$ denote by $P_{M}$ the orthogonal projection onto $M$.

Proposition 6. Let $H$ be a separable infinite-dimensional Hilbert space, and let $\left(e_{1}, e_{2}, \ldots\right)$ be an orthonormal basis in $H$. Let $S \in B(H)$. Then

$$
\|S\|_{e}=\lim _{k \rightarrow \infty}\left\|P_{H_{k}^{\perp}} S P_{H_{k}^{\perp}}\right\|,
$$

where $H_{k}=\bigvee_{j=1}^{k} e_{j}(k \in \mathbb{N})$.

Proof. For each $k \in \mathbb{N}$, the operator $S-P_{H_{k}^{\perp}} S P_{H_{k}^{\perp}}$ is of finite rank, so $\|S\|_{e} \leq\left\|P_{H_{k}^{\perp}} S P_{H_{k}^{\perp}}\right\|$.

Clearly,

$$
\left\|P_{H_{1}^{\perp}} P_{H_{1}^{\perp}}\right\| \geq\left\|P_{H_{2}^{\perp}} S P_{H_{2}^{\perp}}\right\| \geq \cdots,
$$

so the $\operatorname{limit} \lim _{k \rightarrow \infty}\left\|P_{H_{k}^{\perp}} S P_{H_{k}^{\perp}}\right\|$ exists and is greater than or equal to $\|S\|_{e}$.

Suppose on the contrary that $\lim _{k \rightarrow \infty}\left\|P_{H_{k}^{\perp}} S P_{H_{k}^{\perp}}\right\|>\|S\|_{e}$. Then there exists $\varepsilon>0$ such that $\left\|P_{H_{k}^{\perp}} S P_{H_{k}^{\perp}}\right\|>\|S\|_{e}+\varepsilon$ for all $k \in \mathbb{N}$. Find $x_{k} \in H_{k}^{\perp}$, $\left\|x_{k}\right\|=1$ such that $\left\|S x_{k}\right\| \geq\left\|P_{H_{k}} S x_{k}\right\|>\|S\|_{e}+\varepsilon$. Clearly, $x_{k} \rightarrow 0$ weakly. Let $L \in B(H)$ be a compact operator. Then $\left\|L x_{k}\right\| \rightarrow 0$ and $\|S+L\| \geq$ $\sup _{k \in \mathbb{N}}\left\|(S+L) x_{k}\right\| \geq\|S\|_{e}+\varepsilon$. So $\|S\|_{e}=\inf _{L \in \mathcal{K}(H)}\|S+L\| \geq\|S\|_{e}+\varepsilon$, a contradiction.

Corollary 7. Let $H$ be a separable infinite-dimensional Hilbert space, $S \in B(H), t, t^{\prime} \geq 5\|S\|^{2}$. Then:

(i) $\left|\|S+t\|-\left\|S+t^{\prime}\right\|-t+t^{\prime}\right| \leq 4\|S\|^{2} / \min \left\{t, t^{\prime}\right\}$;

(ii) $\left|\|S+t\|_{e}-\left\|S+t^{\prime}\right\|_{e}-t+t^{\prime}\right| \leq 4\|S\|^{2} / \min \left\{t, t^{\prime}\right\}$. 
Proof. Let $x \in H,\|x\|=1$. By Lemma 5 ,

$$
\begin{aligned}
|\|(S+t) x\|-t-\operatorname{Re}\langle S x, x\rangle| & \leq \frac{2\|S\|^{2}}{t}, \\
\left|\left\|\left(S+t^{\prime}\right) x\right\|-t^{\prime}-\operatorname{Re}\langle S x, x\rangle\right| & \leq \frac{2\|S\|^{2}}{t^{\prime}} .
\end{aligned}
$$

So

$$
\left|\|(S+t) x\|-\left\|\left(S+t^{\prime}\right) x\right\|-t+t^{\prime}\right| \leq \frac{4\|S\|^{2}}{\min \left\{t, t^{\prime}\right\}} .
$$

Let $\left(x_{k}\right) \subset H$ be a sequence of unit vectors such that $\left\|(S+t) x_{k}\right\| \rightarrow$ $\|S+t\|$. Without loss of generality we may assume that $\lim _{k \rightarrow \infty}\left\|\left(S+t^{\prime}\right) x_{k}\right\|$ exists. Then

$$
\begin{aligned}
\|S+t\|-\left\|S+t^{\prime}\right\|-t+t^{\prime} & \leq \lim _{k \rightarrow \infty}\left(\left\|(S+t) x_{k}\right\|-\left\|\left(S+t^{\prime}\right) x_{k}\right\|-t+t^{\prime}\right) \\
& \leq \frac{4\|S\|^{2}}{\min \left\{t, t^{\prime}\right\}} .
\end{aligned}
$$

By symmetry, we get (i).

By Proposition 6, we have $\|S+t\|_{e}=\lim _{k \rightarrow \infty}\left\|P_{H_{k}^{\perp}}(S+t) P_{H_{k}^{\perp}}\right\|$, where $H_{k}=\bigvee_{j=1}^{k} e_{j}$ and $\left(e_{1}, e_{2}, \ldots\right)$ is an orthonormal basis in $H$. This together with (i) gives

$$
\begin{aligned}
\mid\|S+t\|_{e}- & \left\|S+t^{\prime}\right\|_{e}-t+t^{\prime} \mid \\
& =\lim _{k \rightarrow \infty}\left|\left\|P_{H_{k}^{\perp}}(S+t) P_{H_{k}^{\perp}}\right\|-\left\|P_{H_{k}^{\perp}}\left(S+t^{\prime}\right) P_{H_{k}^{\perp}}\right\|-t+t^{\prime}\right| \\
& \leq \lim _{k \rightarrow \infty} \frac{4\left\|P_{H_{k}^{\perp}} S P_{H_{k}^{\perp}}\right\|^{2}}{\min \left\{t, t^{\prime}\right\}} \leq \frac{4\|S\|^{2}}{\min \left\{t, t^{\prime}\right\}} . \cdot
\end{aligned}
$$

Lemma 8. Let $T=\left(T_{1}, \ldots, T_{n}\right)$ be an n-tuple of operators on a separable infinite-dimensional Hilbert space $H$. Then there exist mutually orthogonal finite-dimensional subspaces $F_{k} \subset H$ such that $H=\bigoplus_{k=1}^{\infty} F_{k}$ and $P_{r} T_{j} P_{s}=0$ for all $r, s \in \mathbb{N},|r-s| \geq 2$ and $j=1, \ldots, n$ (i.e., the operators $T_{1}, \ldots, T_{n}$ are simultaneously block 3-diagonal).

Proof. Let $\left(e_{1}, e_{2}, \ldots\right)$ be an orthonormal basis in $H$. Let $F_{1}=\bigvee\left\{e_{1}\right\}$. Let $G_{2}=\bigvee\left\{F_{1}, T_{j} F_{1}, T_{j}^{*} F_{1}(1 \leq j \leq n), e_{2}\right\}$ and $F_{2}=G_{2} \ominus F_{1}$. Then $\operatorname{dim} F_{2}<\infty, T_{j} F_{1} \subset F_{1} \oplus F_{2}$ and $T_{j}^{*} F_{1} \subset F_{1} \oplus F_{2}$ for all $j=1, \ldots, n$.

We continue this construction for $k \in \mathbb{N}$ inductively. If $k \geq 3$ and the subspaces $F_{1}, \ldots, F_{k-1}$ have already been constructed, then set

$$
G_{k}=\bigvee\left\{F_{1}, \ldots, F_{k-1}, T_{j} F_{k-1}, T_{j}^{*} F_{k-1}(j=1, \ldots, n), e_{k}\right\}
$$

and $F_{k}=G_{k} \ominus\left(F_{1} \oplus \cdots \oplus F_{k-1}\right)$. Then $\operatorname{dim} F_{k}<\infty, T_{j} F_{k-1} \subset\left(F_{1} \oplus \cdots \oplus F_{k}\right)$ and $T_{j}^{*} F_{k-1} \subset\left(F_{1} \oplus \cdots \oplus F_{k}\right)$ for all $j=1, \ldots, n$. 
If we continue this construction for all $k \in \mathbb{N}$ we get the required decomposition (note that $\bigoplus_{k=1}^{\infty} F_{k}=H$ since $e_{k} \in F_{1} \oplus \cdots \oplus F_{k}$ for each $k$ ).

The following result was proved in [CLSW, Lemma 6]. We formulate it in a more explicit form.

Lemma 9. Let $S \in B(H)$ be block 3-diagonal, i.e., there are finitedimensional subspaces $F_{j}$ such that $H=\bigoplus_{j=1}^{\infty} F_{j}$ and $P_{F_{r}} S P_{F_{s}}=0$ whenever $|r-s| \geq 2$. Denote by $Q_{k}$ the orthogonal projection onto $\bigoplus_{j=k+1}^{\infty} F_{j}$. Let $l, d \in \mathbb{N}, k=l+2 d$ and let $V \in B(H)$ satisfy $V=Q_{k} V Q_{k}$. Then $\|S+V\| \leq \max \left\{\|S\|,\left\|Q_{l}(S+V) Q_{l}\right\|\right\}+\|S\| / \sqrt{d}$.

Proof. Let $x \in H,\|x\|=1$. Then

$$
\begin{array}{r}
\left\|P_{F_{l+1}} x+P_{F_{l+2}} x\right\|^{2}+\left\|P_{F_{l+3}} x+P_{F_{l+4}} x\right\|^{2}+\cdots+\| P_{F_{l+2 d-1}} x+ \\
\quad P_{F_{l+2 d}} x \|^{2} \\
\leq\|x\|^{2}=1 .
\end{array}
$$

So there exists $j_{0}, l+1 \leq j_{0} \leq l+2 d-1 \leq k-1$, such that $\| P_{F_{j_{0}}} x+$ $P_{F_{j_{0}+1}} x \|^{2} \leq d^{-1}$.

Write $x=u+v+w$, where $u \in \bigoplus_{j=1}^{j_{0}-1} F_{j}, v=P_{F_{j_{0}}} x+P_{F_{j_{0}+1}} x$ and $w \in \bigoplus_{j=j_{0}+2}^{\infty} F_{j}$. We have $(S+V) x=S u+S v+(S+V) w$, where $\|S v\| \leq$ $\|S\| \cdot\|v\| \leq\|S\| / \sqrt{d}, S u \in \bigoplus_{j=1}^{j_{0}} F_{j}$ and $(S+V) w=Q_{l}(S+V) Q_{l} w \in$ $\bigoplus_{j_{0}+1}^{\infty} F_{j}$. Thus $S u \perp(S+V) w$ and

$$
\|S u+(S+V) w\| \leq \max \left\{\|S\|,\left\|Q_{l}(S+V) Q_{l}\right\|\right\} .
$$

Hence $\|(S+V) x\| \leq \max \left\{\|S\|,\left\|Q_{l}(S+V) Q_{l}\right\|\right\}+\|S\| / \sqrt{d}$.

Let $T_{1}, \ldots, T_{n} \in B(H)$ be selfadjoint operators. We show that there exist compact selfadjoint operators $K_{1}, \ldots, K_{n} \in B(H)$ such that

$$
\left\|\sum_{j=1}^{n} \alpha_{j} T_{j}+\lambda\right\|_{e}=\left\|\sum_{j=1}^{n} \alpha_{j}\left(T_{j}-K_{j}\right)+\lambda\right\|
$$

for all $\alpha_{1}, \ldots, \alpha_{n}, \lambda \in \mathbb{R}$. Consequently, $W_{e}\left(T_{1}, \ldots, T_{n}\right)=W\left(T_{1}-K_{1}, \ldots\right.$ $\left.\ldots, T_{n}-K_{n}\right)^{-}$.

We show (1) first under the technical assumptions that the space $H$ is separable and $\operatorname{Int}_{\mathbb{R}} W_{e}\left(T_{1}, \ldots, T_{n}\right) \neq \emptyset$, where $\operatorname{Int}_{\mathbb{R}}$ denotes the interior in the sense of $\mathbb{R}^{n}$. However, these assumptions are not necessary.

Let $T=\left(T_{1}, \ldots, T_{n}\right) \in B(H)^{n}$. For $\alpha=\left(\alpha_{1}, \ldots, \alpha_{n}\right)$ write $\|\alpha\|_{1}=$ $\sum_{j=1}^{n}\left|\alpha_{j}\right|$ and $\alpha T=\sum_{j=1}^{n} \alpha_{j} T_{j}$.

THEOREM 10. Let $H$ be a separable infinite-dimensional Hilbert space, let $T=\left(T_{1}, \ldots, T_{n}\right) \in B(H)^{n}$ be an $n$-tuple of selfadjoint operators such that $\operatorname{Int}_{\mathbb{R}} W_{e}(T) \neq \emptyset$. Then there exist compact selfadjoint operators $K_{1}, \ldots, K_{n}$ 
in $B(H)$ such that

$$
\left\|\sum_{j=1}^{n} \alpha_{j} T_{j}+\lambda\right\|_{e}=\left\|\sum_{j=1}^{n} \alpha_{j}\left(T_{j}-K_{j}\right)+\lambda\right\|
$$

for all $\alpha_{1}, \ldots, \alpha_{n}, \lambda \in \mathbb{R}$.

Proof. Without loss of generality we may assume that $\left\|T_{j}\right\|=1$ $(j=1, \ldots, n)$ and $(0, \ldots, 0) \in \operatorname{Int}_{\mathbb{R}} W_{e}(T)$. Let $\varepsilon>0$ be such that $\left(\mu_{1}, \ldots, \mu_{n}\right)$ $\in W_{e}\left(T_{1}, \ldots, T_{n}\right)$ for all $\mu_{1}, \ldots, \mu_{n} \in \mathbb{R},\left|\mu_{j}\right| \leq \varepsilon(j=1, \ldots, n)$.

By Lemma 8, there exist finite-dimensional subspaces $F_{1}, F_{2}, \ldots$ such that $H=\bigoplus_{j=1}^{\infty} F_{j}$ and the operators $T_{1}, \ldots, T_{n}$ are simultaneously block 3 -diagonal with respect to this decomposition. Denote by $Q_{k}$ the orthogonal projection onto the space $\bigoplus_{j=k+1}^{\infty} F_{j}$.

For $m=0,1, \ldots$ we construct inductively $n$-tuples $S^{(m)}=\left(S_{1}^{(m)}, \ldots, S_{n}^{(m)}\right)$ $\in B(H)^{n}$ of selfadjoint operators and an increasing sequence $\left(k_{m}\right)$ of nonnegative integers such that

$$
\begin{aligned}
& Q_{k_{m}} S_{j}^{(m)} Q_{k_{m}}=2^{-m} Q_{k_{m}} T_{j} Q_{k_{m}} \quad(j=1, \ldots, n), \\
& \left\|\sum_{j=1}^{n} \alpha_{j}\left(T_{j}-S_{j}^{(m)}\right)+\lambda\right\| \leq\left\|\sum_{j=1}^{n} \alpha_{j} T_{j}+\lambda\right\|_{e}-\frac{\varepsilon}{2^{m+1}}
\end{aligned}
$$

for all $\alpha_{1}, \ldots, \alpha_{n}, \lambda \in \mathbb{R}, \sum_{j=1}^{n}\left|\alpha_{j}\right|=1$, and

$$
\left\|S_{j}^{(m+1)}-S_{j}^{(m)}\right\| \leq \frac{\left\|T_{j}\right\|}{2^{m+1}} \quad(j=1, \ldots, n) .
$$

For $m=0$ set formally $k_{0}=0$ and $S_{j}^{(0)}=T_{j}(j=1, \ldots, n)$. Clearly, (2) is satisfied.

Let $\alpha_{1}, \ldots, \alpha_{n}, \lambda \in \mathbb{R}, \sum_{j=1}^{n}\left|\alpha_{j}\right|=1$. For $\mu \in \mathbb{R}$ write $\operatorname{sign} \mu=1$ if $\mu \geq 0$ and $\operatorname{sign} \mu=-1$ if $\mu<0$. We have

$$
\left\|\sum_{j=1}^{n} \alpha_{j}\left(T_{j}-S_{j}^{(0)}\right)+\lambda\right\|=|\lambda|
$$

and

$$
\begin{aligned}
\left\|\sum_{j=1}^{n} \alpha_{j} T_{j}+\lambda\right\|_{e} & \geq \max \left\{|\mu|: \mu \in W_{e}\left(\sum_{j=1}^{n} \alpha_{j} T_{j}+\lambda\right)\right\} \\
& \geq \max \left\{\left|\sum_{j=1}^{n} \alpha_{j} \nu_{j}+\lambda\right|:\left(\nu_{1}, \ldots, \nu_{n}\right) \in W_{e}\left(T_{1}, \ldots, T_{n}\right)\right\} \\
& \geq\left|\sum_{j=1}^{n} \frac{\varepsilon \alpha_{j} \operatorname{sign} \lambda}{\operatorname{sign} \alpha_{j}}+\lambda\right|=\varepsilon+|\lambda| .
\end{aligned}
$$


Thus

$$
|\lambda| \leq\left\|\sum_{j=1}^{n} \alpha_{j} T_{j}+\lambda\right\|_{e}-\varepsilon
$$

for all $\alpha_{1}, \ldots, \alpha_{n}, \lambda \in \mathbb{R},\|\alpha\|_{1}=1$. In particular, (3) is satisfied for $m=0$.

Suppose that we have already constructed the $n$-tuples $S^{(0)}, \ldots, S^{(m)} \in$ $B(H)^{n}$ and numbers $k_{0}, \ldots, k_{m} \in \mathbb{N}$ satisfying (2)-(4). Set $r=2^{m+6} / \varepsilon$ and $\delta=\varepsilon / 2^{m+4}$. By Proposition 6 , for all $\alpha \in \mathbb{R}^{n},\|\alpha\|_{1}=1$ and $\lambda \in \mathbb{R},|\lambda| \leq r$, we have

$$
\left\|Q_{k}(\alpha T+\lambda) Q_{k}\right\| \searrow\|\alpha T+\lambda\|_{e}
$$

and the functions $(\alpha, \lambda) \mapsto\left\|Q_{k}(\alpha T+\lambda) Q_{k}\right\|,(\alpha, \lambda) \mapsto\|\alpha T+\lambda\|_{e}$, are continuous. By the Dini theorem the convergence is uniform on the set $A:=\left\{(\alpha, \lambda) \in \mathbb{R}^{n} \times \mathbb{R}:\|\alpha\|_{1}=1,|\lambda| \leq r\right\}$.

Let $l>k_{m}$ satisfy

$$
\left\|Q_{l}(\alpha T+\lambda) Q_{l}\right\|-\|\alpha T+\lambda\|_{e} \leq \delta
$$

for all $(\alpha, \lambda) \in A$. Find $d \in \mathbb{N}$ such that $(r+1) / \sqrt{d} \leq \delta$ and set $k_{m+1}=$ $l+2 d$. For $j=1, \ldots, n$ set

$$
S_{j}^{(m+1)}=S_{j}^{(m)}-\frac{1}{2^{m+1}} Q_{k_{m+1}} T_{j} Q_{k_{m+1}} .
$$

Clearly, $S_{j}^{(m+1)}$ is selfadjoint, $\left\|S_{j}^{(m+1)}-S_{j}^{(m)}\right\| \leq\left\|T_{j}\right\| / 2^{m+1}$ and

$$
\begin{aligned}
Q_{k_{m+1}} S_{j}^{(m+1)} Q_{k_{m+1}} & =2^{-m} Q_{k_{m+1}} T_{j} Q_{k_{m+1}}-2^{-(m+1)} Q_{k_{m+1}} T_{j} Q_{k_{m+1}} \\
& =\frac{1}{2^{m+1}} Q_{k_{m+1}} T_{j} Q_{k_{m+1}} .
\end{aligned}
$$

To show (3), let first $(\alpha, \lambda) \in A$. We have

$$
\begin{aligned}
& \left\|Q_{l}\left(\alpha\left(T-S^{(m+1)}\right)+\lambda\right) Q_{l}\right\| \\
& =\left\|Q_{l}\left(\left(1-2^{-m}\right) \alpha T+\lambda\right) Q_{l}+\frac{1}{2^{m+1}} Q_{k_{m+1}} \alpha T Q_{k_{m+1}}\right\| \\
& \leq \quad\left(1-2^{-m}\right)\left\|Q_{l}(\alpha T+\lambda) Q_{l}\right\|+\frac{1}{2^{m+1}}\left\|Q_{l} \lambda Q_{l}\right\| \\
& \quad+\left\|\frac{1}{2^{m+1}}\left(Q_{l}-Q_{k_{m+1}}\right) \lambda\left(Q_{l}-Q_{k_{m+1}}\right)+\frac{1}{2^{m+1}} Q_{k_{m+1}}(\alpha T+\lambda) Q_{k_{m+1}}\right\| \\
& \leq\left(1-2^{-m}\right)\left(\|\alpha T+\lambda\|_{e}+\delta\right)+\frac{|\lambda|}{2^{m+1}}+\frac{1}{2^{m+1}} \max \left\{|\lambda|,\|\alpha T+\lambda\|_{e}+\delta\right\} \\
& \leq\left(1-\frac{1}{2^{m+1}}\right)\left(\|\alpha T+\lambda\|_{e}+\delta\right)+\frac{1}{2^{m+1}}\left(\|\alpha T+\lambda\|_{e}-\varepsilon\right) \\
& \leq\|\alpha T+\lambda\|_{e}+\delta-\frac{\varepsilon}{2^{m+1}}=\|\alpha T+\lambda\|_{e}-\frac{\varepsilon}{2^{m+1}}+\frac{\varepsilon}{2^{m+4}}
\end{aligned}
$$

(where we used (5)). 
To estimate $\left\|\alpha\left(T-S^{(m+1)}\right)+\lambda\right\|$, we apply Lemma 9 . Define $S=$ $\alpha\left(T-S^{(m)}\right)+\lambda$ and $V=2^{-(m+1)} Q_{k_{m+1}} \alpha T Q_{k_{m+1}}$. By (3), we have $\|S\| \leq$ $\|\alpha T+\lambda\| \leq r+1$. Then

$$
\begin{aligned}
\left\|\alpha\left(T-S^{(m+1)}\right)+\lambda\right\|=\|S+V\| \leq \max \left\{\|S\|,\left\|Q_{l}(S+V) Q_{l}\right\|\right\}+\frac{r+1}{\sqrt{d}} \\
\leq \max \left\{\|\alpha T+\lambda\|_{e}-\frac{\varepsilon}{2^{m+1}},\|\alpha T+\lambda\|_{e}-\frac{\varepsilon}{2^{m+1}}+\frac{\varepsilon}{2^{m+4}}\right\}+\delta \\
=\|\alpha T+\lambda\|_{e}-\frac{\varepsilon}{2^{m+1}}+\frac{\varepsilon}{2^{m+3}} .
\end{aligned}
$$

For $|\lambda|>r$, by Corollary 7 , we have

$$
\begin{aligned}
\|\alpha T+\lambda\|_{e} & -\left\|\alpha\left(T-S^{(m+1)}\right)+\lambda\right\| \\
& =\left\|\frac{\alpha}{\operatorname{sign} \lambda} T+\frac{\lambda}{\operatorname{sign} \lambda}\right\|_{e}-\left\|\frac{\alpha}{\operatorname{sign} \lambda}\left(T-S^{(m+1)}\right)+\frac{\lambda}{\operatorname{sign} \lambda}\right\| \\
& \geq\left\|\frac{\alpha}{\operatorname{sign} \lambda} T+r\right\|_{e}-\left\|\frac{\alpha}{\operatorname{sign} \lambda}\left(T-S^{(m+1)}\right)+r\right\|+\frac{8}{r} \\
& \leq \frac{\varepsilon}{2^{m+1}}-\frac{\varepsilon}{2^{m+3}}+\frac{8}{r}=\frac{\varepsilon}{2^{m+2}} .
\end{aligned}
$$

So $S^{(m+1)}$ satisfies (2)-(4).

Suppose that the $n$-tuples $S^{(m)} \in B(H)^{n}$ have been constructed for all $m \in \mathbb{N}$. For $j=1, \ldots, n$, the sequence $\left(S_{j}^{(m)}\right)_{m}$ is norm convergent; denote by $K_{j}$ its limit. We have

$$
\left\|K_{j}\right\|_{e}=\lim _{m \rightarrow \infty}\left\|S_{j}^{(m)}\right\|_{e} \leq \lim _{m \rightarrow \infty}\left\|Q_{k_{m}} S_{j}^{(m)} Q_{k_{m}}\right\| \leq \lim _{m \rightarrow \infty} 2^{-m}\left\|T_{j}\right\|=0 .
$$

Hence the operator $K_{j}$ is compact for all $j=1, \ldots, n$. Obviously, $K_{j}$ is selfadjoint.

For $\alpha \in \mathbb{R}^{n}, \lambda \in \mathbb{R},\|\alpha\|_{1}=1$ we have

$$
\|\alpha(T-K)+\lambda\|=\lim _{m \rightarrow \infty}\left\|\alpha\left(T-S^{(m)}\right)+\lambda\right\| \leq\|\alpha T+\lambda\|_{e} .
$$

The opposite inequality is clear, so $\|\alpha(T-K)+\lambda\|=\|\alpha T+\lambda\|_{e}$ for all $\alpha \in \mathbb{R}^{n}, \lambda \in \mathbb{R},\|\alpha\|_{1}=1$. Consequently, $\|\alpha(T-K)+\lambda\|=\|\alpha T+\lambda\|_{e}$ for all $\alpha \in \mathbb{R}^{n}, \lambda \in \mathbb{R}$.

Corollary 11. Let $H$ be a separable Hilbert space, let $T=\left(T_{1}, \ldots, T_{n}\right)$ $\in B(H)^{n}$ be an n-tuple of selfadjoint operators. Then there exists an n-tuple $K=\left(K_{1}, \ldots, K_{n}\right) \in B(H)^{n}$ of compact selfadjoint operators such that

$$
\|\alpha T+\lambda\|_{e}=\|\alpha(T-K)+\lambda\|
$$

for all $\alpha \in \mathbb{R}^{n}, \lambda \in \mathbb{R}$.

Proof. We prove the statement by induction on $n$. Let $T=\left(T_{1}, \ldots, T_{n}\right) \in$ $B(H)^{n}$ be an $n$-tuple of selfadjoint operators. 
If $\operatorname{Int}_{\mathbb{R}} W_{e}(T) \neq \emptyset$, then the statement follows from Theorem 10. Suppose on the contrary that $\operatorname{Int}_{\mathbb{R}} W_{e}(T)=\emptyset$.

Since $W_{e}(T)$ is a convex set, there are numbers $\beta_{1}, \ldots, \beta_{n} \in \mathbb{R},\left(\beta_{1}, \ldots, \beta_{n}\right)$ $\neq(0, \ldots, 0)$, such that $\sum_{j=1}^{n} \beta_{j} \nu_{j}=0$ for all $\left(\nu_{1}, \ldots, \nu_{n}\right) \in W_{e}(T)$. So $W_{e}\left(\sum_{j=1}^{n} \beta_{j} T_{j}\right)=\{0\}$. Thus $\left\|\sum_{j=1}^{n} \beta_{j} T_{j}\right\|_{e}=0$ and $L:=\sum_{j=1}^{n} \beta_{j} T_{j}$ is a compact operator. Without loss of generality we may assume that $\beta_{n} \neq 0$. Thus $T_{n}=\beta_{n}^{-1}\left(L-\sum_{j=1}^{n-1} \beta_{j} T_{j}\right)$.

From the induction assumption, there exist selfadjoint operators $K_{1}, \ldots, K_{n-1} \in \mathcal{K}(H)$ such that

$$
\left\|\sum_{j=1}^{n-1} \alpha_{j}\left(T_{j}-K_{j}\right)+\lambda\right\|=\left\|\sum_{j=1}^{n-1} \alpha_{j} T_{j}+\lambda\right\|_{e}
$$

for all $\alpha_{1}, \ldots, \alpha_{n-1}, \lambda \in \mathbb{R}$. Set $K_{n}=\beta_{n}^{-1}\left(L-\sum_{j=1}^{n-1} \beta_{j} K_{j}\right)$. Then $K_{n}$ is a compact selfadjoint operator.

Let $\alpha_{1}, \ldots, \alpha_{n}, \lambda \in \mathbb{R}$. Then

$$
\sum_{j=1}^{n} \alpha_{j}\left(T_{j}-K_{j}\right)=\sum_{j=1}^{n-1} \alpha_{j}\left(T_{j}-K_{j}\right)-\alpha_{n} \beta_{n}^{-1} \sum_{j=1}^{n-1} \beta_{j}\left(T_{j}-K_{j}\right) .
$$

By the induction assumption,

$$
\begin{aligned}
\left\|\sum_{j=1}^{n} \alpha_{j}\left(T_{j}-K_{j}\right)+\lambda\right\| & =\left\|\sum_{j=1}^{n-1} \alpha_{j} T_{j}-\alpha_{n} \beta_{n}^{-1} \sum_{j=1}^{n-1} \beta_{j} T_{j}+\lambda\right\|_{e} \\
& =\left\|\sum_{j=1}^{n} \alpha_{j} T_{j}+\lambda\right\|_{e} \cdot \cdot
\end{aligned}
$$

The assumption of separability is not essential and can be omitted.

TheOREM 12. Let $H$ be an infinite-dimensional Hilbert space, and let $T=\left(T_{1}, \ldots, T_{n}\right) \in B(H)^{n}$ be an $n$-tuple of selfadjoint operators. Then there exists an $n$-tuple $K=\left(K_{1}, \ldots, K_{n}\right) \in B(H)^{n}$ of compact selfadjoint operators such that

$$
\|\alpha T+\lambda\|_{e}=\|\alpha(T-K)+\lambda\|
$$

for all $\alpha \in \mathbb{R}^{n}, \lambda \in \mathbb{R}$.

Proof. There exists a decomposition $H=\bigoplus_{\nu \in J} H^{(\nu)}$ such that all the subspaces $H^{(\nu)}$ are separable and reducing for the operators $T_{1}, \ldots, T_{n}$. Write $T_{j}^{(\nu)}=P_{H^{(\nu)}} T_{j} P_{H^{(\nu)}}$.

For all $\alpha \in \mathbb{R}^{n}, \lambda \in \mathbb{R}$ and $\varepsilon>0$ there are only finitely many $\nu \in J$ such that $\left\|\alpha T^{(\nu)}+\lambda\right\|>\|\alpha T+\lambda\|_{e}+\varepsilon$. So there are only countably many $\nu \in J$ such that $\left\|\alpha T^{(\nu)}+\lambda\right\|>\|\alpha T+\lambda\|_{e}$. Hence there exists a countable subset 
$J_{0} \subset J$ such that $\left\|\alpha T^{(\nu)}+\lambda\right\| \leq\|\alpha T+\lambda\|_{e}$ for all $\nu \notin J_{0}$ and all rational $\alpha_{1}, \ldots, \alpha_{n}, \lambda$. Let $H_{0}=\bigoplus_{\nu \in J_{0}} H^{(\nu)}$. Then $H_{0}$ is a separable subspace reducing for $T_{1}, \ldots, T_{n}$ and $\left\|P_{H \ominus H_{0}}(\alpha T+\lambda) P_{H \ominus H_{0}}\right\| \leq\|\alpha T+\lambda\|_{e}$ for all $\alpha \in \mathbb{R}^{n}, \lambda \in \mathbb{R}$. So we can use Corollary 11 for the operators $P_{H_{0}} T_{j} P_{H_{0}}$.

Corollary 13. Let $T=\left(T_{1}, \ldots, T_{n}\right) \in B(H)$ be an $n$-tuple of selfadjoint operators. Then there exists an $n$-tuple $K=\left(K_{1}, \ldots, K_{n}\right) \in B(H)$ of compact selfadjoint operators such that $W_{e}(T)=\overline{W(T-K)}$. In particular, $\overline{W(T-K)}$ is convex.

Proof. Let $K=\left(K_{1}, \ldots, K_{n}\right) \in B(H)^{n}$ be an $n$-tuple of compact selfadjoint operators such that $\|\alpha(T-K)+\lambda\|=\|\alpha T+\lambda\|_{e}$ for all $\alpha \in \mathbb{R}^{n}, \lambda \in \mathbb{R}$. By Proposition 4,

$$
W_{e}(T)=V(T-K, B(H))=\overline{\operatorname{conv}} W(T-K) \supset \overline{W(T-K)} \supset W_{e}(T) .
$$

So $W_{e}(T)=\overline{W(T-K)}=\overline{\operatorname{conv}} W(T-K)$.

By Remark 3, the same statement is true for an arbitrary $n$-tuple of operators.

Corollary 14. Let $T=\left(T_{1}, \ldots, T_{n}\right) \in B(H)^{n}$. Then there exists an $n$-tuple $K=\left(K_{1}, \ldots, K_{n}\right) \in B(H)$ of compact operators such that $W_{e}(T)=$ $\overline{W(T-K)}$.

We do not know whether Theorem 12 remains true for nonselfadjoint operators.

Problem 15. Let $T \in B(H)^{n}$. Does there exist $K \in \mathcal{K}(H)^{n}$ such that

$$
\|\alpha T+\lambda\|_{e}=\|\alpha(T-K)+\lambda\|
$$

for all $\alpha \in \mathbb{C}^{n}, \lambda \in \mathbb{C}$ ?

Using the method of Theorem 10, it is possible to obtain a positive answer if $\operatorname{Int} W_{e}(T) \neq \emptyset$. However, there are technical problems if $W_{e}(T)$ is a set of a lower dimension.

3. Olsen's problem. The method from the previous section can also be used to improve the results of C. L. Olsen [O].

Theorem 16. Let $S \in B(H), n \in \mathbb{N}$ and $\left\|S^{n}\right\|_{e} \neq 0$. Then there exists a compact operator $K \in B(H)$ such that $\left\|(S-K)^{j}\right\|=\left\|S^{j}\right\|_{e}$ for $j=1, \ldots, n$.

Proof. Without loss of generality we may assume that $\|S\|=1$. Furthermore, we may assume that the space $H$ is separable (cf. proof of Theorem 12).

By Lemma 8, there exist finite-dimensional subspaces $F_{1}, F_{2}, \ldots$ such that $H=\bigoplus_{j=1}^{\infty} F_{j}$ and the operators $S, S^{2}, \ldots, S^{n}$ are block 3-diagonal with respect to this decomposition. Denote by $Q_{k}$ the orthogonal projection onto the space $\bigoplus_{j=k+1}^{\infty} F_{j}$. 
Let $0<\varepsilon<\left\|S^{n}\right\|_{e} / 2$. For $1 \leq j \leq n$ we have $2 \varepsilon \leq\left\|S^{n}\right\|_{e} \leq\left\|S^{j}\right\|_{e}$. $\left\|S^{n-j}\right\|_{e} \leq\left\|S^{j}\right\|_{e}$. Let $c=2^{n} \varepsilon^{-1}$. For $m=0,1, \ldots$ set $s_{m}=c /(c+m)$. So $s_{0}=1, s_{m} \searrow 0$ and

$$
s_{m}-s_{m+1}=\frac{c}{(c+m)(c+m+1)} \leq \frac{s_{m}}{c}=\frac{s_{m} \varepsilon}{2^{n}} .
$$

We construct inductively operators $S_{m} \in B(H)$ and an increasing sequence $\left(k_{m}\right)$ of nonnegative integers such that:

$$
\begin{aligned}
& Q_{k_{m}} S_{m} Q_{k_{m}}=\left(1-s_{m}\right) Q_{k_{m}} S Q_{k_{m}}, \\
& \left\|S_{m+1}-S_{m}\right\| \leq s_{m}-s_{m+1}, \\
& \left\|S_{m}^{j}\right\| \leq\left\|S^{j}\right\|_{e}-\varepsilon s_{m} \quad(j=1, \ldots, n) .
\end{aligned}
$$

For $m=0$ set $S_{0}=0$ and $k_{0}=0$. Then clearly (6) and (8) are satisfied.

Let $m \geq 0$ and suppose that $S_{m}$ and $k_{m}$ have already been constructed. Choose a positive number $\delta<\min \left\{\left(s_{m}-s_{m+1}\right) \varepsilon, s_{m} \varepsilon 2^{-n}\right\}$.

By Proposition 6, there exists $l>k_{m}$ such that

$$
\left\|Q_{l} S^{j} Q_{l}\right\| \leq\left\|S^{j}\right\|_{e}+\delta \quad(j=1, \ldots, n) .
$$

Let $k_{m+1}>l+2 / \delta^{2}+2$. Set

$$
S_{m+1}=S_{m}+\left(s_{m}-s_{m+1}\right) Q_{k_{m+1}} S Q_{k_{m+1}} .
$$

Clearly (6) and (7) are satisfied.

To show (8), fix $j \in\{1, \ldots, n\}$. Write $W=\left(s_{m}-s_{m+1}\right) Q_{k_{m+1}} S Q_{k_{m+1}}$ and $V=S_{m+1}^{j}-S_{m}^{j}=\left(S_{m}+W\right)^{j}-S_{m}^{j}$. So $V$ can be expressed as the sum of $2^{j}-1$ operators, each being a product of $j$ elements of the pair $\left\{S_{m}, W\right\}$, where $W$ appears at least once. Since $\|W\| \leq s_{m}-s_{m+1}$ and $\left\|S_{m}\right\| \leq 1$ by (8), we have $\|V\| \leq\left(2^{j}-1\right)\left(s_{m}-s_{m+1}\right)$.

By Lemma $9,\left\|S_{m+1}^{j}\right\| \leq \max \left\{\left\|S_{m}^{j}\right\|,\left\|Q_{l} S_{m+1}^{j} Q_{l}\right\|\right\}+\delta$, where $\left\|S_{m}^{j}\right\| \leq$ $\left\|S^{j}\right\|_{e}-\varepsilon s_{m}$ by the induction assumption. Further,

$$
\begin{aligned}
\left\|Q_{l} S_{m+1}^{j} Q_{l}\right\| & \leq\left\|Q_{l} S_{m}^{j} Q_{l}\right\|+\|V\|=\left(1-s_{m}\right)^{j}\left\|Q_{l} S^{j} Q_{l}\right\|+\|V\| \\
& \leq\left(1-s_{m}\right)\left(\left\|S^{j}\right\|_{e}+\delta\right)+\left(2^{j}-1\right)\left(s_{m}-s_{m+1}\right) \\
& \leq\left(1-s_{m}\right)\left\|S^{j}\right\|_{e}+\delta+\frac{\left(2^{n}-1\right) s_{m} \varepsilon}{2^{n}} \\
& \leq\left\|S^{j}\right\|_{e}+s_{m}\left(-\left\|S^{j}\right\|_{e}+\varepsilon\right) \leq\left\|S^{j}\right\|_{e}-\varepsilon s_{m} .
\end{aligned}
$$

Hence $\left\|S_{m+1}^{j}\right\| \leq\left\|S^{j}\right\|_{e}-\varepsilon s_{m}+\delta \leq\left\|S^{j}\right\|_{e}-\varepsilon s_{m+1}$.

Suppose that we have constructed operators $S_{m}$ and integers $k_{m}$ satisfying (6)-(8). Then the sequence $\left(S_{m}\right)$ is norm-convergent. Denote its limit 
by $U$. For $j=1, \ldots, n$ we have $\left\|U^{j}\right\|=\lim _{m \rightarrow \infty}\left\|S_{m}^{j}\right\| \leq\left\|S^{j}\right\|_{e}$. Further,

$$
\begin{aligned}
\|S-U\|_{e} & =\lim _{m \rightarrow \infty}\left\|Q_{k_{m}}(S-U) Q_{k_{m}}\right\| \\
& \leq \lim _{m \rightarrow \infty}\left(\left\|Q_{k_{m}}\left(S-S_{m}\right) Q_{k_{m}}\right\|+\left\|Q_{k_{m}}\left(S_{m}-U\right) Q_{k_{m}}\right\|\right) \\
& \leq \lim _{m \rightarrow \infty}\left(s_{m}+\left\|S_{m}-U\right\|\right)=0 .
\end{aligned}
$$

So the operator $K:=S-U$ is compact and we have $\left\|(S-K)^{j}\right\|=\left\|U^{j}\right\|=$ $\left\|S^{j}\right\|_{e}$ for all $j=1, \ldots, n$.

Corollary 17. Let $S \in B(H), n \in \mathbb{N}$. Then there exists a compact operator $K \in B(H)$ such that $\left\|(S-K)^{n}\right\|=\left\|S^{n}\right\|_{e}$.

Proof. If $\left\|S^{n}\right\|_{e} \neq 0$ then the statement was proved in the previous theorem. If $\left\|S^{n}\right\|_{e}=0$ then the statement was proved in [OP].

Acknowledgements. The author wishes to thank to M. Mbekhta and M. González for drawing his attention to the paper [CLSW] and for fruitful discussions about this and similar problems.

The research was supported by grants No. 201/09/0473 of GA ČR, IAA100190903 of GA AV and by IRP AV OZ 10190503.

\section{References}

[B] M. Barraa, Essential numerical range of elementary operators, Proc. Amer. Math. Soc. 133 (2005), 1723-1726.

[BD1] F. F. Bonsall and J. Duncan, Numerical Ranges of Operators on Normed Spaces and of Elements of Normed Algebras, London Math. Soc. Lecture Note Ser. 2, Cambridge Univ. Press, London, 1971.

[BD2] - - , Numerical Ranges II, London Math. Soc. Lecture Note Ser. 10, Cambridge Univ. Press, London, 1973.

[CLSW] C. K. Chui, D. A. Legg, R. R. Smith and J. D. Ward, On a question of Olsen concerning compact perturbations of operators, Michigan Math. J. 24 (1977), 119-127.

[CSSW] C. K. Chui, P. W. Smith, R. R. Smith and J. D. Ward, L-ideals and numerical range preservation, Illinois J. Math. 21 (1977), 365-373.

[FSW] P. A. Fillmore, J. G. Stampfli and J. P. Williams, On the essential numerical range, the essential spectrum, and a problem of Halmos, Acta Sci. Math. (Szeged) 33 (1972), 179-192.

[LP] C. K. Li and Y. T. Poon, The joint essential numerical range of operators: convexity and related results, Studia Math. 194 (2009), 91-104.

[O] C. L. Olsen, Norms of compact perturbations of operators, Pacific J. Math. 68 (1977), 209-228.

[OP] C. L. Olsen and J. K. Plastiras, Quasialgebraic operators, compact perturbations, and the essential norm, Michigan Math. J. 21 (1974), 385-397.

[S] J. G. Stampfli, Compact perturbations, normal eigenvalues and a problem of Salinas, J. London Math. Soc. 2 (1974), 165-175. 
[TC] M. Takaguchi and M. Chō, The joint numerical range and the joint essential numerical range, Sci. Rep. Hirosaki Univ. 27 (1980), 6-8.

Vladimír Müller

Mathematical Institute

Czech Academy of Sciences

Žitna 25, 11567 Praha 1, Czech Republic

E-mail: muller@math.cas.cz 